\title{
Late and early time phenomenology of Higgs-dependent cutoff
}

\section{F. Bezrukov} \\ Arnold Sommerfeld Center for Theoretical Physics, \\ Department für Physik, Ludwig-Maximilians-Universität München, \\ Institute for Nuclear Research of the Russian Academy of Sciences, \\ 60th October Anniversary prospect 7a, Moscow 117312, Russia \\ E-mail: Fedor.Bezrukov@physik.uni-muenchen.de
}

\section{Gorbunov}

Institute for Nuclear Research of the Russian Academy of Sciences, 60th October Anniversary prospect 7a, Moscow 117312, Russia

E-mail: gorby@ms2.inr.ac.ru

\section{Shaposhnikov}

\author{
Institut de Théorie des Phénomènes Physiques, \\ École Polytechnique Fédérale de Lausanne, \\ CH-1015 Lausanne, Switzerland \\ E-mail: Mikhail.Shaposhnikov@epfl.ch
}

\begin{abstract}
The analysis of theories with non-minimal coupling of Higgs field to gravity revealed that they enter into strong coupling regime above certain Higgs-dependent cutoff, which may be considerably below the Planck scale. Assuming that the effective theory, complementing the Standard Model or its minimal extension - the $\nu \mathrm{MSM}$ - contains a set of higher dimensional operators suppressed by the Higgs-dependent cutoff, we analyse the reheating of the Universe after the Higgs inflation. We show that extra terms do not spoil the Higgs inflation, but can lead to baryogenesis and to warm sterile neutrino dark matter production at the reheating stage of the Universe expansion. They can also result in neutrino mass generation and proton decay.
\end{abstract}

KEYwORDS: inflation, higher dimensional operators, physics of the early universe, baryogenesys, dark matter. 


\section{Contents}

1. Introduction 1

2. Prerequisite discussion: Higgs-inflation framework and higher dimensional operators 4

3. Higher dimensional operators in the SM 7

3.1 Role of the non-renormalizable operators in low energy (present day) physics 7

3.2 Neutrino reheating via dimension-5 operators 8

3.3 Lepton asymmetry in the Early Universe from 5-dimensional operator $\quad 10$

4. Higher dimensional operators in the $\nu \mathrm{MSM}$ and dark matter sterile neutrinos

$\begin{array}{lll}\text { 4.1 Stability at cosmological time-scales } & 14\end{array}$

$\begin{array}{lll}4.2 & \text { Dark matter production } & 15\end{array}$

$\begin{array}{ll}\text { 5. Summary and conclusions } & 16\end{array}$

A. Generation of fermions by varying mass term, scalar gravity sector cutoff case

\section{Introduction}

The Universe is flat, homogeneous and isotropic, and has the primordial density perturbations with almost scale-invariant spectrum. ${ }^{1}$ All these above may be well explained by a hypothetical inflationary stage of the Early Universe before it became hot. This stage can be realized within the Standard Model (SM) of particle physics extended by non-minimal coupling of the SM Higgs field $H$ to gravity [1]

$$
\mathcal{L}_{\text {gravity }}=\frac{M_{P}^{2}}{2} R+\xi H^{\dagger} H R
$$

where $M_{P} \equiv 1 / \sqrt{8 \pi G_{N}}=2.44 \times 10^{18} \mathrm{GeV}$ is the reduced Planck mass. In [2] (see also [3]) we analyzed the history of the Universe within the Higgs inflation scenario in two theories, assuming their validity up to the Planck scale. The first one was the Standard Model and the second one was the $\nu$ MSM (an extension of the SM by 3 singlet fermions

\footnotetext{
${ }^{1}$ It is well known for a long time, e.g.: «Чудище обло, озорно, огромно с тризевной и Лаей,» В. Tредиаковский, Телемахида (1766).
} 
with masses below the electroweak scale, for a review see ref. [4]). One of our main goals in [2] was to determine the initial conditions for the hot Big Bang. In other words, we addressed the question whether the quantum numbers, effectively conserved in the SM (such as $B-L$, where $B$ and $L$ are baryon and lepton numbers respectively) and in the $\nu \mathrm{MSM}$ (primordial abundances of the singlet leptons) can be generated during inflation or reheating of the Universe. For this end we added to the SM and to the $\nu$ MSM higher dimensional operators, breaking these conservation laws, and suppressed by the scale $\zeta M_{P}$. The bounds on parameter $\zeta$ were derived from requirement that the Higgs inflation is not spoiled. The constraints on $\zeta$ allowed to estimate the $B-L$ excess and the abundances of singlet fermions after reheating. We found that the contribution of higher dimensional operators, bounded in this way, to the production of Warm Dark Matter (WDM) and to baryogenesis is negligible. We concluded, therefore, that the production of sterile neutrino $\mathrm{WDM}^{2}$ and baryon asymmetry of the Universe must be a low-temperature phenomenon, having nothing to do with inflation and reheating.

The aim of the present paper is to revisit this analysis in connection with new results obtained recently in the investigations of consistency of Higgs inflation. We present below a short account of this progress, to motivate our present study.

For successful inflation yielding primordial density perturbations with the amplitude matched to the cosmological observations [5], it is required at the tree level that the nonminimal coupling $\xi$ must be rather large,

$$
\xi \simeq 47000 \sqrt{\lambda}
$$

where $\lambda$ is the Higgs field self-coupling constant. This relation is modified by loop corrections, see $[6,7,8,9]$ for numerical results and [10] for further discussion. ${ }^{3}$

The requirement $\xi \gg 1$ raised doubts in the validity of the Higgs inflation [11, 12]. The estimate of the high energy scattering amplitudes in the low energy electroweak vacuum showed that the theory enters into a strong-coupling regime (the tree unitarity is broken) at energies $E$ well below the Planck scale, $E \simeq M_{P} / \xi$. Since this energy is much smaller than the values of the Higgs field during inflation, $H \sim M_{P} / \sqrt{\xi}$, the authors of the papers $[11,12]$ concluded that the Higgs inflation is an "unnatural" phenomenon, as it intrinsically requires fine-tuning of the Higgs potential at large values of the Higgs field.

However, the analysis [10] (see also [13]) revealed that the theory remains in a weak coupling regime below certain cutoff scale $\Lambda(h)$, which in general depends on the Higgs field and is in fact above the inflationary scale. This makes the Higgs inflation self-consistent and "natural" $[10,13]$. However, the phenomenology and predictivity of the model depend on the properties of the UV-complete theory at high energies [10]. Here we analyze the post-

\footnotetext{
${ }^{2}$ On the contrary, a sterile neutrino with the mass exceeding $100 \mathrm{keV}$ (a Cold Dark Matter candidate) can be created during the reheating stage of the Universe in the necessary amount.

${ }^{3}$ Everywhere in this paper, except for the Section 3.1, we will need the values of the constants at the inflationary scale. Then, this relation is hardly modified compared to the tree level result, and the only subtlety is the connection of the Higgs self-coupling at high scale with the physical Higgs mass. Under the assumptions, formulated in ref. [10], this relation is quite precisely given by the SM renormalization group running, see Fig. 5 of ref. [7].
} 
inflationary phenomenology using the effective field theory parametrization of the effects which might come from the high energy theory.

One can distinguish several cutoff scales corresponding to violation of perturbative unitarity in the different sectors of the model. In the gravity-scalar sector the cutoff is ${ }^{4}$

$$
\Lambda_{g-s}(h) \simeq \begin{cases}\frac{M_{P}}{\xi}, & \text { for } h \lesssim \frac{M_{P}}{\xi}, \\ \frac{\xi h^{2}}{M_{P}}, & \text { for } \frac{M_{P}}{\xi} \lesssim \lesssim \frac{M_{P}}{\sqrt{\xi}} \\ \sqrt{\xi} h, & \text { for } h \gtrsim \frac{M_{P}}{\sqrt{\xi}}\end{cases}
$$

The cutoff for purely gravitational interactions (graviton scattering off graviton) is just the effective Planck scale (see eq. (1.1))

$$
\Lambda_{\text {Planck }}^{2} \simeq M_{P}^{2}+\xi h^{2} .
$$

The cutoff associated with SM gauge interactions at $h>\frac{M_{P}}{\xi}$ is

$$
\Lambda_{\text {gauge }} \simeq h
$$

and coincides with $\frac{M_{P}}{\xi}$ at smaller fields $h$.
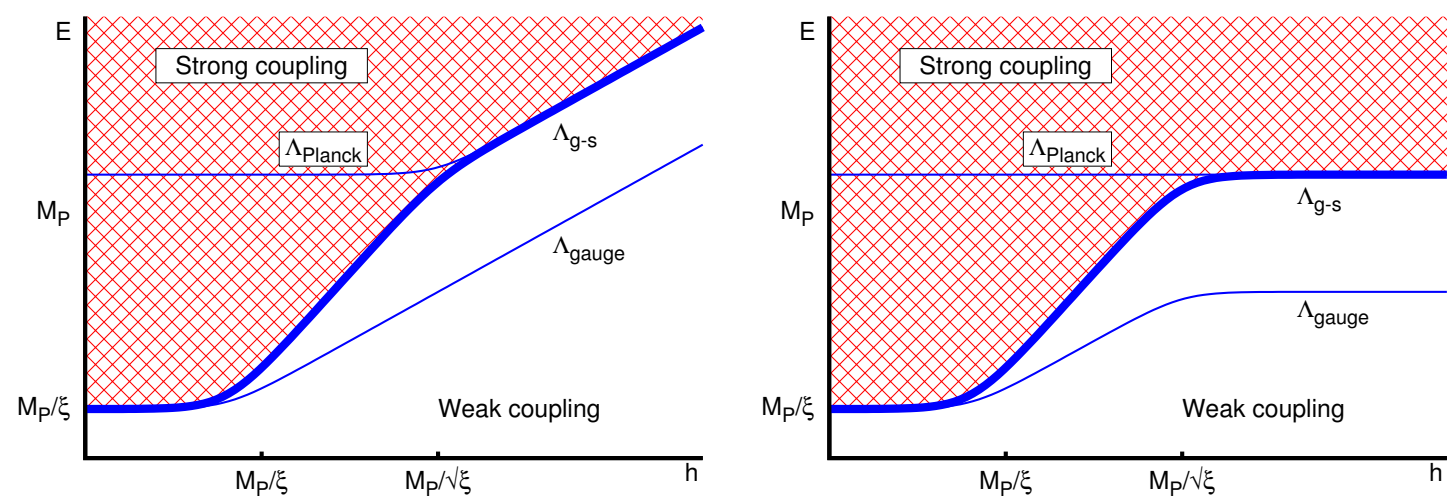

Figure 1: Schematic depiction of the cut-offs (1.3), (1.4), and (1.5) in the Jordan (left) and Einstein (right) frames.

One of the reasons the inflation takes place while the theory remains in weakly coupling regime is because it does not involve large momentum transfers. The validity of the theory above momenta $\Lambda(h)$ is unclear. Thus, the question arises what happens when momentum transfers $E$ are between $\Lambda_{\text {gauge }}(h)$ and $\Lambda_{\text {Planck. }}$. Remaining within the minimal setup, ${ }^{5}$ we can imagine two scenarios:

\footnotetext{
${ }^{4}$ We present all cutoffs in the Jordan frame, corresponding to eq. (1.1) and use the unitary gauge, $H^{T}=(0, h / \sqrt{2})$.

${ }^{5}$ Alternatively, it is possible to attempt perturbative UV completion by introducing new states at the cut-off scale [14].
} 
1. The Lagrangian of gravity + SM (actually, its viable and phenomenologically complete extension such as the $\nu \mathrm{MSM}$ ) remains valid up to the Planck scale (without any higher order operators suppressed by lower scales), but the system at some $h$ and large momenta $\Lambda_{\text {gauge }}(h)<E<\Lambda_{\text {Planck }}$ is in the strong coupling regime and requires non-perturbative methods for its description. Essentially this point of view was taken in our previous studies of inflation and reheating of the Universe $[1,2,6,7]$.

2. The consistency of the theory in this range of energies requires introduction (below the cut-off scale) of higher order operators, suppressed by a Higgs-dependent cutoff $\Lambda(h)$

The present paper is devoted to the study of phenomenology of the second scenario in the SM and $\nu \mathrm{MSM}$, as the first one was considered in [2]. The fact that the cutoff is considerably smaller than the Planck scale may induce a number of interesting effects at low energies, such as proton decay or neutrino mass generation. In the early Universe, the baryon and lepton number violating reactions are greatly enhanced in comparison with the first scenario, which can potentially change baryogenesis or leptogenesis. The sterile neutrino WDM primordial production at the reheating stage after inflation may rise considerably in the second scenario. At the same time, even with the small, but Higgs-dependent cutoff, the inflation is not modified.

The paper is organized as follows. In Section 2 we discuss higher-dimensional operators which could emerge in the model with Higgs-dependent cutoff (1.3) and explain why they do not spoil the Higgs inflation. The case of the SM is considered in Section 3. We study lowenergy phenomenology and reconsider the processes in post-inflationary Universe. We show that higher-dimensional operators may produce lepton asymmetry and hence be responsible for the matter-over-antimatter domination in the present Universe. We explore the possible role of higher-dimensional operator in reheating. Section 4 is entirely devoted to the $\nu$ MSM and we address here all the questions we do in Section 3. We show, in addition, that the model new fields - sterile neutrinos - might form warm dark matter, and describe new options for reheating and leptogenesis. We summarize our results in Section 5, concluding with discussion of UV-completion of the model with regard to cosmology.

\section{Prerequisite discussion: Higgs-inflation framework and higher dimen- sional operators}

We start with introduction of relevant framework and short review on the relevant facts about the Universe evolution in the model with the non-minimally coupled Higgs inflation following refs. $[1,2]$.

From now on all the computations in the paper we are going to make in the Einstein frame, which is connected to the Jordan frame (1.1) by the conformal transformation

$$
g_{\mu \nu} \rightarrow \hat{g}_{\mu \nu}=\Omega^{2} g_{\mu \nu}, \quad \Omega^{2}(h)=\frac{M^{2}+\xi h^{2}}{M_{P}^{2}} .
$$


This change induces modification of the kinetic term of the scalar field, which can be returned to canonical form by the field redefinition

$$
\chi \simeq \begin{cases}h & \text { for } \quad h<X_{\mathrm{cr}} \equiv \sqrt{\frac{2}{3}} \frac{M_{P}}{\xi}, \\ \sqrt{\frac{3}{2}} M_{P} \log \Omega^{2}(h) & \text { for } \quad h>X_{\mathrm{cr}} \equiv \sqrt{\frac{2}{3}} \frac{M_{P}}{\xi} .\end{cases}
$$

The action (in the Einstein frame) then becomes an action of the usual minimally coupled to gravity scalar field $\chi$ with the potential

$$
U(\chi)=\frac{1}{\Omega^{4}[h(\chi)]} \frac{\lambda}{4}\left[h^{2}(\chi)-v^{2}\right]^{2} .
$$

The rest of the action (after proper rescaling of the fermion fields) looks like the usual SM action in the unitary gauge with proper rescaling of the mass terms by $\Omega[h(\chi)]$. I.e. the usual SM mass terms for fermion $\psi$ and vector boson $A_{\mu}$ get the general form of

$$
\mathcal{L}_{\text {Yukawa }}=\frac{Y h(\chi)}{\Omega[h(\chi)]} \bar{\psi} \psi, \quad \mathcal{L}_{\text {gauge }}=\frac{g^{2} h^{2}(\chi)}{2 \Omega^{2}[h(\chi)]} A_{\mu} A^{\mu},
$$

with $Y$ and $g$ being the corresponding Yukawa and gauge coupling constants.

The potential (2.3) provides for slow roll inflation in the region $h>M_{P} / \sqrt{\xi}(\chi>$ $M_{P}$ ), and gives rise to primordial density perturbations of observed amplitude for model parameters obeying (1.2). For small field values $h<M_{P} / \xi$ and equally small energies the model behaves as the usual SM: this is the usual thermal evolution of the Universe.

In the intermediate region $M_{P} / \xi<h<M_{P} / \sqrt{\xi}$ the scalar potential is approximately quadratic. Oscillations of the scalar field with frequency

$$
\omega \equiv \sqrt{\frac{\lambda}{3}} \frac{M_{P}}{\xi}
$$

and amplitude in the intermediate region above reheat the Universe. In the absence of the higher dimensional operators suppressed by Higgs-dependent cutoff the reheating happens via production of gauge and Higgs bosons by the oscillating field background as described in $[2,3]$, and the reheating temperature $T_{r}$ is confined in the range

$$
1.4 \times 10^{-5} M_{P}<T_{r}<4.5 \times 10^{-5}\left(\frac{\lambda}{0.25}\right)^{1 / 4} M_{P},
$$

where the relation (1.2) is put in.

The overall conformal factor $\Omega(h)$ becomes relevant only for $h>M_{P} / \sqrt{\xi}$, which corresponds to the inflationary regime. This means, that after inflation (starting from the post-inflationary reheating) the value of the cut-off in the Jordan and Einstein frames are the same. As far as the current work deals mostly with the post inflationary processes, the only change we need to make to get to the Einstein frame with canonically normalized fields is the variable change (2.2).

Let us proceed with the discussion on higher dimensional operators. As far as the full UV complete theory for the non-minimally coupled Higgs inflation is not yet developed, 
one can not make any definite predictions. Meanwhile we can explore what happens if various higher dimensional operators are added to the model.

We will analyze the effect of the following higher dimensional terms

$$
\begin{aligned}
\delta \mathcal{L}_{\mathrm{NR}}= & -\frac{a_{6}}{\Lambda^{2}}\left(H^{\dagger} H\right)^{3}+\cdots \\
& +\frac{\beta_{L}}{4 \Lambda} F_{\alpha \beta} \bar{L}_{\alpha} \tilde{H} H^{\dagger} L_{\beta}^{c}+\frac{\beta_{B}}{\Lambda^{2}} O_{\text {baryon violating }}+\cdots+\text { h.c. } \\
& +\frac{\beta_{N}}{2 \Lambda} H^{\dagger} H \bar{N}^{c} N+\frac{b_{L_{\alpha}}}{\Lambda} \bar{L}_{\alpha}(\not D N)^{c} \tilde{H}+\cdots
\end{aligned}
$$

where $L_{\alpha}$ are SM leptonic doublets, $\alpha=1,2,3, N$ stands for right handed sterile neutrinos potentially present in the model, $\tilde{H}_{a}=\epsilon_{a b} H_{b}^{*}, a, b=1,2$; here $\Lambda$ depends on the background (Higgs) field value, $\Lambda=\Lambda(h)$.

In [2] the limits on these operators were obtained in the assumption that the cut-off scale is constant. Then these operators either directly change the inflationary potential, like the Higgs potential (2.7a), either generate the contributions to the potential by loop corrections. For example, from the first two operators in (2.7) contributions in the Jordan frame are $h^{6} / \Lambda^{2}$ and $h^{8} / \Lambda^{4} \log \left(m^{2}(h) / \mu^{2}\right)$. Both terms grow much faster than $h^{4}$ and can not be compensated by the fourth power of the conformal factor, thus leading to non-flat potential and bad inflationary properties. Numerical estimate [2] shows, that the minimal value of constant cut-off, consistent with inflationary predictions, is $\Lambda_{\text {const }} \sim M_{P}$. However, all the choices (1.3), (1.4), and (1.5) grow proportionally to $h$ in the inflationary regime, and the dangerous terms in the potential behave just as $h^{4}$ for inflation. Then after conformal transformation the shape of the inflationary potential (2.3) remains the same, with modified constants. This means, that the inflationary predictions for the initial density perturbations remain unchanged, and the only modification is a correction to the normalization (1.2) of the non-minimal coupling.

Perturbativity up to energy scale $\Lambda$ implies that the numerical coefficients $a, \beta$ are of order one or less. With the lack of knowledge about underlying theory, this is the only requirement on higher dimensional terms (2.7). However, one can distinguish three different situations.

First, it could be that still unknown dynamics at the scale $\Lambda \ll M_{P}$ does not violate itself any global symmetries of the SM. Then, numerical coefficients in front of corresponding symmetry-violating operators are suppressed, so that the effective energy scale entering, say, baryon number violating operators in (2.7) is the Plank mass or even higher. The opposite case is when all gauge invariant operators enter (2.7) with numerical coefficients of order one. Finally, the intermediate situation corresponds to some hierarchy in these coefficients. As we observe below, this third situation is the most interesting, as such models exhibit the richest phenomenology. In particular, we will discuss in due course the case where at the scale $\Lambda$ higher order operators could dominate over similar ones of lower order. In particular, for the operators of the form

$$
\delta \mathcal{L}^{\tau}=y_{\tau} L_{\tau} H E_{\tau}+\beta_{y} L_{\tau} H E_{\tau} \frac{H^{\dagger} H}{\Lambda^{2}}+\cdots
$$


one can fancy the hierarchy

$$
1 \sim \beta_{y} \gg y_{\tau} \sim 10^{-2} .
$$

For small $H$ this gives nothing new. However, during reheating we can get $H \sim \Lambda$, so the second term in (2.8) can overcome the first one at large values of the Higgs field, and make a significant contribution compared to the lowest order estimate. Note, that hierarchy (2.9) does not generally imply strong coupling below the Plank scale. To simplify further discussion we suppose that all similar higher order operators are negligible.

Note in passing that in this work we treat a particular set of couplings $\beta, a_{6}, \ldots$ from pure phenomenological point of view without any relation to what happens to the operators (2.7) above the scale $\Lambda$ and without any discussion of matching conditions at critical values of the Higgs field (1.3). Having in mind a possible UV-completion like asymptotic safety [15] we also disregard all dangerous contributions naively expected in the theory (2.7) with a formal high-energy cutoff at the scale $\Lambda$, such as quadratic in $\Lambda$ corrections to the Higgs boson mass and others.

\section{Higher dimensional operators in the SM}

\subsection{Role of the non-renormalizable operators in low energy (present day) physics}

At present time the Higgs field dependence of the cutoff in the (2.7) is not observable, since the Higgs background has relaxed to its vacuum value $h=v=246 \mathrm{GeV}$. So the effect of the higher dimensional operators (2.7) can be analyzed as usual, and is most important for the lowest values of the cutoff, that is $M_{P} / \xi$, see eqs. (1.3)-(1.5) and the nearby discussion. As far as the operators are suppressed by this scale, the experimentally interesting effects can be expected from the operators violating symmetries of the renormalizable SM action. These are the operators in (2.7b) responsible for the violation of lepton and baryon numbers.

The lowest order non-renormalizable lepton number violating operator (first term in $(2.7 \mathrm{~b})$ ) provides the Majorana mass terms for active neutrinos

$$
\mathcal{L}_{\nu \nu}^{(5)}=\frac{\beta_{L} v^{2}}{4 \Lambda} \frac{F_{\alpha \beta}}{2} \bar{\nu}_{\alpha} \nu_{\beta}^{c}+\text { h.c. . }
$$

To explain the observed pattern of the active neutrino masses the scale of this lepton number violating operator should not be higher than

$$
\Lambda<2.8 \times 10^{14} \mathrm{GeV} \times \beta_{L} \times\left(\frac{3 \times 10^{-3} \mathrm{eV}^{2}}{\Delta m_{\mathrm{atm}}^{2}}\right)^{1 / 2} .
$$

For the allowed range of parameter $\xi$ at the electroweak scale (see [7], Fig. 4 for discussion and relation to the Higgs boson mass), $\xi \simeq 10^{3}-10^{5}$, we get for the scale $\Lambda$

$$
\Lambda=\frac{M_{P}}{\xi} \sim(0.3-35) \times 10^{14} \mathrm{GeV} .
$$

The neutrino masses can be explained without taking too large parameter $\beta$, namely with

$$
\beta_{L} \simeq 0.1-10
$$


The next operator, important at low energies, is the baryon number violating one, as it leads to the proton decay. We can use the estimate of the proton decay in the standard $S U(5)$ model to place a lower limit on the scale $\Lambda$. Indeed, for the $S U(5)$ case the proton life-time is [16] (the exact form of the relevant dimension- 6 operators contributing to the decay is given in the reference)

$$
\tau_{p \rightarrow \pi^{0} e^{+}}=1.0 \times 10^{35} \text { years } \times\left(\frac{1 / 25}{g_{5}^{2} / 4 \pi}\right)^{2}\left(\frac{M_{V}}{10^{16} \mathrm{GeV}}\right)^{4},
$$

where $M_{V}=g_{5} v_{5} / 2$, and $v_{5}$ is the vev responsible for spontaneous breaking of $S U(5)$ gauge symmetry. Thus, current limit on the proton decay, $\tau_{p \rightarrow \pi^{0} e^{+}}>1.6 \times 10^{33}$ years [17], yields a bound on the scale $v_{5}$, which in our case corresponds to the scale $\Lambda$ so that

$$
\Lambda \gtrsim \sqrt{\beta_{B}} \times 10^{16} \mathrm{GeV} \times\left(\frac{\tau_{p \rightarrow \pi^{0} e^{+}}}{1.6 \times 10^{33} \text { years }}\right)^{1 / 4} .
$$

The discrepancy between the limits (3.2) and (3.6) is about 40 (for $\beta_{B}=\beta_{L}=1$ ) and can be reconciled only with a particular hierarchy between the dimensionless coefficients:

$$
\beta_{L} \gtrsim 36 \times \sqrt{\beta_{B}}
$$

For example, if $\beta_{L} \sim 1$ within the interval (3.4), one has to chose $\beta_{B} \lesssim 10^{-3}$. Thus, baryon number violating scale cannot be $\Lambda=M_{P} / \xi$, it is certainly higher.

This implies, that while the neutrino masses can be explained for a wide range of the Higgs masses, if the lepton number is violated at the scale (1.5) or (1.3), the proton decay should be prevented by some means. If allowed, its rate must be suppressed by a higher scale, like gravity scale (1.4). The required hierarchy between $\beta_{L}$ and $\sqrt{\beta_{B}}$ though large, still may be accidental; then it suggests that proton decay has to be observed in the nearest future experiments. Otherwise, the hierarchy may be a natural outcome of some unknown mechanism operating at the scale of UV-completion. Indeed, for instance, the two operator under discussion (2.7b) differ in a particular respect: one involves the Higgs fields, while another does not. A specific renormalization flow evolution of the Higgs-containing operators might be responsible for establishing the required hierarchy (3.7) at the scale $M_{P} / \xi$

\subsection{Neutrino reheating via dimension-5 operators}

It was shown in [2], that if the suppression scale equals the Planck mass, then the particle production by the higher dimensional operators is negligible, and hence their impact on the reheating. However, this is not immediately obvious with the Higgs-field dependent cut-off. Let us analyze the contribution to reheating of the lepton number violating operator in (2.7a).

Scalar gravity sector cutoff. We start from the operator (3.1), and omit the numerical coefficients $F_{\alpha \beta} \sim 1$ in further estimates. During the preheating, while the background 
field evolves according to eq. (3.4) of [2], we have the following effective time-dependent Majorana type mass source for the active neutrinos

$$
m(t) \simeq \begin{cases}\frac{\beta_{L}}{4} \sqrt{\frac{3}{\lambda}} \omega, & \text { for } \chi(t) \gtrsim \sqrt{\frac{3}{\lambda}} \omega, \\ \frac{\beta_{L}}{4} \sqrt{\frac{\lambda}{3}} \frac{1}{\omega} \chi^{2}, & \text { for } \chi(t) \lesssim \sqrt{\frac{3}{\lambda}} \omega\end{cases}
$$

While the number of generated fermions of given 3 -momentum $\mathbf{k}$ is small, $n_{\mathbf{k}} \ll 1$, the effect of this can be treated by first order perturbation theory. The calculation described in Appendix A yields for the fermion density saturated by the moment of reheating

$$
n=\beta_{L}^{2} \frac{\sqrt{\lambda}}{16 \sqrt{3} \pi^{2}} \xi X_{\mathrm{cr}}^{3}
$$

Without a hierarchy in $F_{\alpha \beta}$ the total number density of all three species of active neutrinos is roughly trice the amount of (3.9). To check, whether this leads to preheating, we should compare the energy density in neutrinos with that in the inflaton oscillating with amplitude $X$, see Appendix A for details. The equality between these two culminates the reheating, and the corresponding reheating temperature would be

$$
T_{r} \sim\left(\frac{10 \lambda}{27 g_{*}}\right)^{1 / 4} \frac{\beta_{L}}{2 \pi \sqrt{2 \pi}} \frac{M_{P}}{\sqrt{\xi}} .
$$

Though it is parametrically larger than the temperature of reheating due to renormalizable operators [2], $T_{r}^{\text {renorm }} \propto \frac{M_{P}}{\xi}$, but numerically, for $\beta_{L} \sim 1$, it is of the same order, cf. (2.6)

$$
T_{r} \simeq 1.7 \times \beta_{L} \times 10^{14} \mathrm{GeV} \sim T_{r}^{\text {renorm }} \simeq 10^{14} \mathrm{GeV} .
$$

Hence, higher order operators do not significantly enhance reheating.

We check in Appendix A that the Pauli blocking is relevant for $\beta_{L} \sim 1$ if the reheating temperature is not at the lower end of the allowed range (2.6). This diminishes the possible contribution of higher dimensional operators to reheating: they are never really relevant to this process.

Gauge sector cutoff. One could think, that with the lower cut-off (1.5) the contribution to reheating becomes significant. However, with more active generation Pauli blocking effects would suppress the process much earlier, so the contribution of the generated neutrinos to the energy balance during reheating can not really exceed calculations in the previous section. Of course, as before, one would expect that still existence of additional reheating channel somewhat favors the higher end of the reheating temperature interval $(2.6)$.

Finally, we conclude, that the estimate (2.6) is still a valid one, with additional preference for the higher temperature part of the allowed region. 


\subsection{Lepton asymmetry in the Early Universe from 5-dimensional operator}

The next interesting effect which can appear from the lepton violating operators in (2.7) is the generation of the baryon asymmetry via leptogenesis.

One can ask whether it is possible to generate a reasonably large lepton asymmetry $\Delta n_{L} \equiv n_{L}-n_{\bar{L}}$ with the nonrenormalizable operator (2.7b) at the post-inflationary preheating stage. At a given time the amount of asymmetry is determined by averaging of lepton number operator $\hat{Q}_{L}$, which evolves in accordance with the Heisenberg equation,

$$
i \frac{d}{d t} \hat{Q}_{L}=\left[\hat{H}_{\mathrm{int}}, \hat{Q}_{L}\right] .
$$

Here the interaction Hamiltonian comprises both SM interactions and all nonrenormalizable terms (2.7). From these equations one finds in a usual way (see, e.g. [18]), that at a given time the production rate of lepton asymmetry, $d \Delta n_{L} / d t$ is proportional to the imaginary part of the minimal nontrivial trace of the SM Yukawa couplings $Y_{\alpha \beta}=Y_{\alpha} \cdot \delta_{\alpha \beta}$ entering

$$
\mathcal{L}_{Y}=-Y_{\alpha} \bar{L}_{\alpha} H E_{\alpha}+\text { h.c. },
$$

and to the couplings $F_{\alpha \beta}$ entering $(2.7 \mathrm{~b})$. This trace reads

$$
\beta_{L}^{4} \operatorname{Tr}\left(F F^{\dagger} F Y Y F^{\dagger} Y Y\right)
$$

and, since the Yukawa constants $Y_{\alpha \beta}$ can be chosen to be diagonal, the dominant term is

$$
\beta_{L}^{4} Y_{3}^{4} F_{3 \beta} F_{\alpha \beta}^{*} F_{\alpha 3} F_{33}^{*}
$$

where $Y_{3}=y_{\tau}$ obviously provides with the largest contribution. For simplicity we adopt the unitary gauge while making an estimate of the generated asymmetry. The Majorana terms (2.7b) connect only neutrinos, while the Dirac terms (3.11), instead, connect electrically charged leptons. Thus, to get the term (3.12) from a corresponding fermionic loop one has to insert the electroweak vertices with $W$-bosons everywhere between the vertices proportional to $Y$ and $F$ (overall four electroweak vertices). These $W$-bosons can be either virtual or external. The former case implies 3-loop contributions, while the latter case actually describes scattering of $W$-bosons produced in the early Universe via Higgs field oscillations as explained in ref. [2].

Let us consider the 3-loop contribution, which is numerically suppressed by the loop factor

$$
\left(\frac{1}{4 \pi^{2}}\right)^{3} \sim 10^{-5}
$$

During the reheating the relevant energies for the generation asymmetry are given by the Higgs field oscillation frequency $\omega$, which is much smaller than the gauge boson mass at this epoch, see (2.4), (2.5)

$$
\omega \equiv \sqrt{\frac{\lambda}{3}} \frac{M_{P}}{\xi} \ll M_{W} \equiv g \sqrt{\frac{M_{P}|\chi|}{2 \sqrt{6} \xi}} .
$$


Thus, $W$-bosons can be actually integrated out which gives the effective Fermi lagrangian with the following combination playing the role of the squared Fermi constant (see eq. (3.8) in [2])

$$
\frac{g^{4}}{M_{W}^{4}} \sim \frac{\xi^{2}}{M_{P}^{2} \chi^{2}} \sim \frac{1}{h^{4}}
$$

The couplings (2.7b) and (3.11) yield the Higgs-dependent factor (see eq. (3.9) in [2])

$$
\frac{\chi^{2} M_{P}^{2}}{\xi^{2}} \cdot\left(\frac{\chi M_{P}}{\xi \Lambda}\right)^{4} \sim h^{4}\left(\frac{h^{2}}{\Lambda}\right)^{4} .
$$

Collecting the factors (3.14), (3.13), (3.15), and (3.16) we get for the asymmetry generated during one oscillation of the Higgs field

$$
\Delta n_{L}^{(1)} \sim \omega^{-1} \cdot\left(\frac{1}{4 \pi^{2}}\right)^{3} \cdot\left(\frac{m_{\tau}}{v}\right)^{4} \cdot \frac{\omega^{4}}{\lambda^{2}} \cdot\left(\frac{\chi}{\Lambda}\right)^{4} \beta_{L}^{4} \sim \omega^{-1}\left(\frac{1}{4 \pi^{2}}\right)^{3} y_{\tau}^{4} \beta_{L}^{4} h^{4}\left(\frac{h}{\Lambda}\right)^{4} .
$$

Let us now estimate the result for the possible choices of the high-energy cutoff scale.

Gauge cutoff. The maximal effect is expected from the lowest available cutoff, the gauge one (1.5). In this case the ratio $h / \Lambda$ goes to one, and asymmetry generation rate scales as $\Delta n_{L}^{(1)} \propto h^{4} \propto \chi^{2}$. The amplitude $X$ of the oscillations of the field $\chi$ is inversely proportional to the cosmological time. This means, that integration of the asymmetry over a period of matter dominated expansion corresponds to multiplication by the time of expansion (or number of oscillations). Recall, we found in Section 3.2 that reheating develops here almost as it does without any higher dimensional operators. For reheating culminating at the amplitude of the Higgs field oscillations $X_{r}$ (see [2]) the number of field oscillations during reheating is $N \sim \xi X_{\mathrm{cr}} / X_{r}$, and the entropy after reheating is $s \sim g_{*}^{1 / 4} \lambda^{3 / 4}\left(X_{r} X_{\mathrm{cr}}\right)^{3 / 2}$. Collecting this we get for the lepton asymmetry

$$
\Delta_{L} \simeq \frac{\Delta n_{L}^{(1)} N}{s} \sim \beta_{L}^{4} y_{\tau}^{4}\left(\frac{1}{4 \pi^{2}}\right)^{3} \frac{1}{g_{*}^{1 / 4} \lambda^{5 / 4}} \xi\left(\frac{X_{\mathrm{cr}}}{X_{r}}\right)^{1 / 2} .
$$

For the estimated in [2] range of reheating, caused by lower order operators, eq. (3.17) gives the asymmetry to be in the range

$$
\beta_{L}^{4}\left(\frac{y_{\tau}}{0.01}\right)^{4}\left(\frac{0.25}{\lambda}\right)^{7 / 4} \times 10^{-15} \times \xi<\Delta_{L}<\beta_{L}^{4}\left(\frac{y_{\tau}}{0.01}\right)^{4}\left(\frac{0.25}{\lambda}\right)^{3 / 2} \times 5 \times 10^{-15} \times \xi,
$$

or, inserting relation (1.2),

$$
\beta_{L}^{4}\left(\frac{y_{\tau}}{0.01}\right)^{4}\left(\frac{0.25}{\lambda}\right)^{5 / 4} \times 10^{-10}<\Delta_{L}<\beta_{L}^{4}\left(\frac{y_{\tau}}{0.01}\right)^{4}\left(\frac{0.25}{\lambda}\right) \times 10^{-9},
$$

where the smaller value corresponds to the larger reheating temperature. One can see, that if $\beta_{L} \sim 1$ this may be just on the very edge of reasonable value. In particular, for the ranges (3.18), (3.4) one can both explain the active neutrino masses and generate a sufficient amount of lepton asymmetry at preheating stage. However, the result is rather 
sensitive to the higher dimensional operators in the SM lepton sector (2.8). As far as during reheating $h \sim \Lambda$, all the higher order operators in (2.8) are not automatically suppressed compared to the leading one. This means, that just the largest one contributes (assuming that the whole sum still behaves nicely). Thus, for the example of hierarchy (2.9) the result (3.17) is enhanced by a factor $\left(\beta_{y} / y_{\tau}\right)^{4}$ leading to increase in the net lepton asymmetry up to 8 orders of magnitude. In this case quite large amount of lepton asymmetry can be produced. Note, that the higher order operators enhancing the lepton asymmetry generation are obviously irrelevant for lepton number violating processes at low energies discussed in Section 3.1 and hence phenomenologically viable.

Gravity-scalar sector cutoff. Otherwise, if we adopt the gravity-scalar sector cutoff (1.3), which is the smallest one available, then the ratio $h^{2} / \Lambda$ goes to $M_{P} / \xi$. We are left with $\Delta n_{L}^{(1)} \propto$ const, so in the expanding Universe the asymmetry is saturated at lower times, that is at the reheating. Integration of the asymmetry over a period of matter dominated expansion corresponds ${ }^{6}$ to multiplication of $\Delta n_{L}^{(1)}$ by the total number of oscillations. Then, for reheating which happened at the amplitude of the Higgs field oscillations $X_{r}$ (see [2]), it took approximately $N \sim \xi X_{\mathrm{cr}} / X_{r}$ oscillations and lead to the entropy after reheating $s \sim g_{*}^{1 / 4} \lambda^{3 / 4}\left(X_{r} X_{\mathrm{cr}}\right)^{3 / 2}$. Collecting all the relevant factors we get for the lepton asymmetry

$$
\Delta_{L} \simeq \frac{\Delta n_{L}^{(1)} N}{s} \sim \frac{\Delta n_{L}^{(1)}}{g_{*}^{1 / 4} \lambda^{3 / 4}} \frac{\xi}{X_{\mathrm{cr}}^{3}}\left(\frac{X_{\mathrm{cr}}}{X_{r}}\right)^{5 / 2} \sim \beta_{L}^{4} Y_{\tau}^{4}\left(\frac{1}{4 \pi^{2}}\right)^{3} \frac{2 \sqrt{2}}{3 g_{*}^{1 / 4} \lambda^{5 / 4}} \xi\left(\frac{X_{\mathrm{cr}}}{X_{r}}\right)^{5 / 2} .
$$

For the estimated in [2] range of reheating, caused by lower order operators, this means asymmetry in the range

$$
\beta_{L}^{4}\left(\frac{0.25}{\lambda}\right)^{15 / 4} \times 2.7 \times 10^{-17} \xi<\Delta_{L}<\beta_{L}^{4}\left(\frac{0.25}{\lambda}\right)^{5 / 2} \times 10^{-14} \xi,
$$

or adopting (1.2)

$$
\beta_{L}^{4}\left(\frac{0.25}{\lambda}\right)^{13 / 4} \times 6.3 \times 10^{-13}<\Delta_{L}<\beta_{L}^{4}\left(\frac{0.25}{\lambda}\right)^{2} \times 2.4 \times 10^{-10}
$$

where smaller value corresponds to larger reheating temperature. One can see, that if $\beta_{L} \sim 1$, this is too small asymmetry. However, the result is rather sensitive to the higher dimensional operators in the SM lepton sector (2.8). By the end of reheating we have $H^{\dagger} H / \Lambda^{2} \simeq 3 X_{\mathrm{cr}} / 4 X_{r}$ and all the operators in (2.8) contribute proportional to their coupling constants. Thus, dimension-6 operator (2.8) with hierarchy (2.9) still leads to enhancement, but not that large as with the gauge cutoff, and decreasing with higher reheating temperatures. The result (3.17) is enhanced by a factor $\left(3 \beta_{y} X_{\mathrm{cr}} / 4 y_{\tau} X_{r}\right)^{4}$ leading to increase in the net lepton asymmetry: lower and upper limits of (3.20) grow by factors of 15 and $2 \times 10^{5}$, respectively. We should keep in mind, that the higher value of the

\footnotetext{
${ }^{6}$ With accuracy of a factor of order one, which depends also on the treatment of field-dependent cutoff in the calculation.
} 
reheating temperature is more realistic (especially with account of additional contributions from neutrino reheating described in Section 3.2), and at high reheating temperatures even this enhancement does not lead to viable leptogenesis. So, generation of sufficient baryon asymmetry with scalar-gravity cutoff is possible, but may be problematic, unless $\beta_{L}$ takes highest values in the interesting interval (3.4).

Finally, at later stages of the Universe evolution the first term in (2.7b) gives rise to lepton violating scattering in plasma [19], which might wash out the lepton asymmetry generated at preheating. To clear up the situation we adopt formulas from ref. [20] for our case of $\nu_{\tau}$ playing the major role in lepton asymmetry generation. With parameters in front of the first term in (2.7b) yielding the correct values of masses and mixing angles in neutrino sector we found that the lepton violating scatterings are slower than the Universe expansion rate at $T \gtrsim 10^{14} \mathrm{GeV}$ and hence are out of equilibrium. This temperature is about the reheating temperature of the model (2.6), and the washing out processes (if any) are not efficient. At later times the sphaleron processes come into equilibrium and transfer the lepton asymmetry generated at preheating to the net baryon asymmetry [21] $\Delta_{B} \approx$ $\Delta_{L} / 3$. From the estimates above, (3.18), (3.20) we conclude that the baryon asymmetry of the Universe can be explained with higher dimensional operators, if suppressed by the gauge cut-off (1.5), and with some hierarchy like (2.9) this conclusion becomes certain. In the case of gravity-scalar cutoff (1.3) the hierarchy like (2.9) is definitely required in order to produce the sufficiently large amount of the baryon asymmetry. Moreover, the reheating temperature in this case has not to be too high, otherwise another mechanism of baryogenesis has to be implemented in the model to explain the observed matter-overantimatter asymmetry of the Universe.

Note in passing that in principle additional operators in quark sector can also contribute in a similar way to the generation of the baryon asymmetry, but these operators are proportional to higher powers of $H^{\dagger} H / \Lambda^{2}$ and the generic analysis is rather involved.

We conclude this Section by the statement that with higher order operators in Higgsinflation there are possibilities to explain both the neutrino oscillations and baryon asymmetry of the Universe.

\section{Higher dimensional operators in the $\nu \mathrm{MSM}$ and dark matter sterile neutrinos}

DM problem needs introduction of some new particles in the SM. A minimal example of such physics is $\nu$ MSM $[22,4]$, the Standard Model extension with three sterile neutrinos $N_{I}, I=1,2,3$, lighter than electroweak bosons. To the SM Lagrangian the following terms are added

$$
\delta L^{\nu \mathrm{MSM}}=i \bar{N}_{I} \partial_{\mu} \gamma^{\mu} N_{I}+\frac{1}{2} M_{I} \bar{N}_{I}^{c} N_{I}-f_{\alpha I} \bar{L}_{\alpha} N_{I} \tilde{H}+\text { h.c. }
$$

The lightest sterile neutrino, say mostly $N_{1}$, comprises dark matter while the two heavier sterile neutrinos due to nonzero Yukawa couplings $f_{\alpha I} \ll 1$ provide active neutrino with 
masses. Sterile neutrinos can contribute to the dark matter and lepton asymmetry generation. One of the important difficulties in the plain $\nu \mathrm{MSM}$ setup is the generation of correct abundance of the DM sterile neutrino (see [4] for a review), which requires very severe fine tuning in sterile neutrino sector [23] or a special source of dark matter neutrino in the early Universe, e.g. decaying inflaton [24, 25]. We consider in this Section whether the higher order operators (2.7) can change the situation.

The sterile neutrino has renormalizable Yukawa interactions (4.1) with the fields of the SM. These interactions inevitably give rise to the decays of $N$ such as $N \rightarrow 3 \nu$ and $N \rightarrow \gamma \nu$, and to its production in the early Universe. The dark matter candidate in the form of sterile neutrino was introduced in [26] (for a review see [4] and references therein). The analysis of the non-resonant production of neutrinos and the X-ray constrains puts an upper limit on its mass, $M<4 \mathrm{keV}$, which happens to be in conflict with the lower limits on from Ly- $\alpha$ analysis $M>8 \mathrm{keV} .^{7}$

We will see, that the higher-dimensional operators can change the situation and lead to efficient production of this DM candidate, consistent with other constraints. Still, even in this case the mass of sterile neutrino should be relatively small, as is argued below.

\subsection{Stability at cosmological time-scales}

The assumption that all higher dimensional operators are included is very powerful as it allows to conclude generally that any DM candidates have to be unstable (though very long-lived) and relatively light, so that the best strategy for their search is searches for emerging in dark matter decay photons of $\mathrm{MeV}$ or lower energies. Considering $\nu \mathrm{MSM}$ as an example, the argument above goes as follows.

Even if all couplings $f_{\alpha 1}$ in (4.1) are zero, they will be generated by five-dimensional operators, proportional to $b_{L_{\alpha}}$ in (2.7). The use of equations of motion for $N$ leads to an estimate of effective Yukawa coupling

$$
f_{\alpha 1} \sim b_{L_{\alpha}} \frac{M_{1}}{\Lambda} .
$$

So, $N_{1}$ is unstable due to higher dimensional operators, with the $\gamma \nu$ partial width of the order

$$
\Gamma_{N_{1} \rightarrow \gamma \nu} \sim \frac{9 b_{L_{\alpha}}^{2} \alpha G_{F}^{2}}{512 \pi^{4}} \frac{v^{2} M_{1}^{5}}{\Lambda^{2}} .
$$

Taking a constraint from EGRET data $\tau_{\gamma \nu} \gtrsim 10^{27} \mathrm{~s}$ [30], we obtain that $M_{1} \lesssim 200 \mathrm{MeV}$ for $b_{L_{\alpha}} \sim 1$ and $\Lambda=M_{P}$. Similar bounds follow from five-dimensional operators like the second term in $(2.7 \mathrm{c})$ where derivatives act on lepton doublets. With smaller scale $\Lambda=M_{P} / \xi$ instead of $M_{P}$ in this term (or larger than (4.2) Yukawa couplings), the upper limit on sterile neutrino mass becomes stronger,

$$
M_{1} \lesssim 4 \mathrm{MeV}
$$

\footnotetext{
${ }^{7}$ The resonant production of DM sterile neutrinos [27, 28], which occurs in the presence of lepton asymmetry, escapes these constraints [29].
} 
It is also worth mentioning that light dark matter sterile neutrino can not help to explain the active neutrino oscillations, as it contributes only a tiny amount to sterile neutrino mass via seesaw mechanism in (4.1); to gain active neutrino masses, heavier unstable neutrinos are required, which however can be searched for in particle physics experiments [31].

\subsection{Dark matter production}

The sterile neutrino $N_{1}$ can be generated from the dimension 5 operator in (2.7) during the hot stage and at the preheating stage.

Production at hot stage. The coupling, proportional to $\beta_{N}$ in (2.7), produces sterile neutrinos in the primordial plasma at hot stage of the Universe evolution. If stable at cosmological time scales this sterile neutrino is a viable candidate for dark matter. If it never came to equilibrium, its mass is limited from above at a given $\beta_{N} / \Lambda$ by imposing $\Omega_{N} \lesssim \Omega_{D M}$. This limit can be read off from eq. (5.20) of ref. [2] after multiplying the l.h.s. of that equation by $4 \Lambda^{2} / M_{P}^{2}$ and choosing $T_{r}=\Lambda$ and $\beta=\beta_{N}$. This gives

$$
M_{N} \lesssim 215 \mathrm{keV} \frac{\Lambda}{\beta_{N}^{2} M_{P}}
$$

Equality in the equation above saturates if sterile neutrinos comprise right amount of the dark matter.

We observe that with $\Lambda \sim M_{P}$ and $\beta_{N} \sim 1$ the dark matter neutrino has mass in sub-MeV region. The lower the scale $\Lambda$, the lighter the dark matter neutrino. With low energy cutoff $\Lambda=M_{P} / \xi$ one obtains

$$
M_{N} \lesssim 10 \times\left(\frac{0.03}{\beta_{N}}\right)^{2} \mathrm{keV}
$$

Neutrino average momentum is close to the thermal average momentum in the primordial plasma, so the constraints from Ly- $\alpha$ discussed in [4] are relevant in our case either. Thus, for the cutoff $\Lambda=M_{P} / \xi$ the viable dark matter implies $\beta_{N} \lesssim 0.03$, sterile neutrino might form warm dark matter. If $\beta_{N} \sim 1$ and $\Lambda=M_{P} / \xi$, stable at cosmological time scales sterile neutrinos contribute to the hot dark matter component, and hence can not solve the dark matter problem. With $\beta_{N} \sim 1, \Lambda=M_{P}$ the sterile neutrinos are cold and can solve the problem. Note, that a particular value of dark matter mass $M_{N}$ should be corrected if higher order operators change somewhat the reheating in the model, as we discuss in Section 3.2.

However, we will see now, that at the preheating stage the neutrino production is much more active.

Dark Matter generation during preheating with dimension-5 operator suppressed by $\Lambda(h)$. Let us consider the sterile neutrino production by the same dimension 5 operator as that in previous Section, suppressed by the Higgs-dependent scale, corresponding to the gravity-scalar cutoff (1.3),

$$
\mathcal{L}_{\text {int }}=\beta_{N} \frac{H^{\dagger} H}{2 \Lambda} \bar{N}^{c} N=\frac{\beta_{N}}{4} \frac{h^{2}}{\Lambda(h)} \bar{N}^{c} N .
$$


Calculation proceeds along the same lines as in Section 3.2. The sterile neutrino density at late times is given by eq. (A.7) with obvious replacement $\beta_{L} \rightarrow \beta_{N}$.

Dividing this density by the entropy at reheating we get for sterile neutrino abundance

$$
\begin{aligned}
\Delta_{N}(X)=\frac{90}{g_{*} 4 \pi^{2}} \beta_{N}^{2} \frac{\sqrt{\lambda}}{2 \sqrt{3} \pi^{2}} \xi \frac{X_{\mathrm{cr}}^{3}}{T_{r}^{3}} & \\
& =\frac{90}{g_{*} 4 \pi^{2}} \beta_{N}^{2} \frac{\sqrt{\lambda}}{\sqrt{3} \pi^{2}} \frac{2^{3 / 2}}{3^{3 / 2} \xi^{2}} \frac{M_{P}^{3}}{T_{r}^{3}}=1.7 \times 10^{-4} \beta_{N}^{2} \sqrt{\frac{\lambda}{0.25}} \frac{1}{\xi^{2}} \frac{M_{P}^{3}}{T_{r}^{3}}
\end{aligned}
$$

(seemingly large $\xi$ suppression is not there, as it is compensated by two extra powers of $\left.M_{p} / T_{r}\right)$. Thus, we get for $\Omega_{D M}=0.223$

$$
\frac{\Omega_{N}}{\Omega_{D M}}=\frac{\beta_{N}^{2} M_{N}}{2.4 \mathrm{keV}} \sqrt{\frac{\lambda}{0.25}} \frac{1}{\xi^{2}} \frac{M_{P}^{3}}{T_{r}^{3}}=\beta_{N}^{2} \frac{M_{N}}{1.3 \times 10^{-4} \mathrm{keV}} \sqrt{\frac{0.25}{\lambda}}\left(\frac{1.1 \times 10^{14} \mathrm{GeV}}{T_{r}}\right)^{3} .
$$

We can see, that if the constant $\beta_{N}>10^{-2}$, the sterile neutrino DM should be lighter than $\mathrm{keV}$, which violates the structure formation bounds. Thus, if $\beta_{N} \sim 1$ it is a problem to generate DM sterile neutrino with the higher dimensional operators; however with hierarchy $\beta_{N} \lesssim 10^{-2}$ it is still possible. Obviously, the case of gauge cutoff leads to even larger production.

The case of the Planck mass cutoff (1.4) coincides with the analysis in [2], which also allows successful generation of the DM density

$$
\frac{\Omega_{N}}{\Omega_{D M}}=\beta_{N}^{2} \frac{M_{N}}{10^{4} \mathrm{keV}}\left(\frac{0.25}{\lambda}\right)\left(\frac{1.1 \times 10^{14} \mathrm{GeV}}{T_{r}}\right) .
$$

In this case the sterile neutrino should be rather heavy, and additional care is needed to assure its stability (see previous section).

\section{Summary and conclusions}

We have analyzed various effects that can appear from the higher order operators in Higgs inflationary theory. In the previous analysis [2] we used additional operators suppressed by a constant (field independent) cutoff, which lead to the conclusion that either the inflationary properties of the theory are spoiled, or no significant generation of any chemical potentials is possible at preheating due to these operators.

In this paper we analyzed a wider class of operators, suppressed by the Higgs dependent cut-off, which is essential to sustain good properties of the theory [10]. The key observation here is that now the cut-off can be lowered significantly at present time and at reheating without spoiling the inflationary properties. As a result, with addition of properly chosen higher dimensional operators one can explain many effects. With proper tuning of the coefficients in front of these operators, one can explain neutrino masses, obtain proton decay, generate baryon asymmetry and sterile neutrino DM (within $\nu$ MSM framework). However, all this is only possible with rather specific choices of the hierarchies between the higher dimensional operators, and a generic prediction seems to be impossible. 
The work is supported in part by the grant of the President of the Russian Federation NS-5525.2010.2 (government contract 02.740.11.0244). D.G. thanks ITPP EPFL for hospitality. The work of F.B. is partially supported by the Humboldt foundation. The work of D.G. is supported in part by FAE program (government contract ח520), RFBR grants 11-02-92108-ЯФ_a, 11-02-01528-a, and by SCOPES program. The work of M.S. is supported by the Swiss National Science Foundation.

\section{A. Generation of fermions by varying mass term, scalar gravity sector cutoff case}

Let us analyze the generation of fermions by the operators $(2.7 \mathrm{~b})$ or $(2.7 \mathrm{c})$. In the presence of the time varying Higgs field background this leads to the Hamiltonian for the fermions

$$
\hat{H}_{\text {int }} \equiv \int d^{3} \mathbf{x} m(t) \bar{\Psi} \Psi
$$

with the time varying mass (3.8). The analysis of the generation of the fermions, while the amount of generated particles is small (occupation numbers much smaller than unity) can be done perturbatively and closely follows appendix $\mathrm{F}$ of [2]). We treat the constant mass $m_{0}=\beta_{L} \sqrt{\frac{3}{\lambda}} \omega / 4$ as a part of the free Hamiltonian. Then the number density at the moment t

$$
n_{k}(t)=\int_{0}^{t}\left(m\left(t^{\prime}\right)-m_{0}\right) \mathrm{e}^{2 i k_{0} t^{\prime}} d t^{\prime} \int_{0}^{t}\left(m\left(t^{\prime \prime}\right)-m_{0}\right) \mathrm{e}^{-2 i k_{0} t^{\prime \prime}} d t^{\prime \prime} .
$$

and total particle number

$$
n(t)=\int \frac{d^{3} \mathbf{k}}{(2 \pi)^{3}} n_{k}(t) .
$$

Integration is the same as in [2], leading to

$$
n_{\mathbf{k}}\left(t_{l}\right)=\frac{1}{16} \frac{\sin ^{2}\left(2 l \frac{\pi k_{0}}{\omega}\right)}{\sin ^{2}\left(\frac{\pi k_{0}}{\omega}\right)} L^{2}
$$

where the constant is slightly changed:

$$
L=\beta_{L} \sqrt{\frac{\lambda}{3}} \frac{1}{4 \omega} X^{2} \cdot \frac{\omega}{k_{0}}\left\{\frac{1}{k_{0}+\omega} \sin \left[4 \pi\left(\frac{k_{0}}{\omega}+1\right) \epsilon\right]-\frac{1}{k_{0}-\omega} \sin \left[4 \pi\left(\frac{k_{0}}{\omega}-1\right) \epsilon\right]\right\},
$$

where we removed $2 k_{0}$ from the denominator and changed $3 \lambda X^{2} \rightarrow \beta_{L} \sqrt{\frac{\lambda}{3}} \frac{1}{4 \omega} X^{2}$ (compare (3.8) and (C.1) of [2]); the ratio $\omega / k_{0}$ should always stay, because it is the ratio of the source frequency and the energy $k_{0}$ in the oscillating exponent. The parameter $\epsilon$ is defined as

$$
\sin (2 \pi \epsilon) \equiv \sqrt{\frac{3}{\lambda}} \frac{\omega}{X}
$$

At large times the ratio leads to the sum of delta functions over momenta, and integration over momenta gives

$$
\frac{n(t)}{t}=\beta_{L}^{2} \frac{\lambda}{48} X^{4} \frac{1}{(2 \pi)^{3}} \sum_{l=2}^{\infty} \sqrt{1-\frac{1}{l^{2}}} \cdot\left(\frac{1}{l+1} \sin [4 \pi(l+1) \epsilon]-\frac{1}{l-1} \sin [4 \pi(l-1) \epsilon]\right)^{2} .
$$


Summation for small $\epsilon$ can be performed using the integral (cf. appendix E of [2])

$$
S_{3}=\int_{0}^{\infty}(4 \pi \epsilon)^{3} \frac{d u}{u^{4}}(2 u \cos u-2 \sin u)^{2}=\frac{2 \pi}{3}(4 \pi \epsilon)^{3} .
$$

This finally yields the generation rate (we reintroduced also the expansion of the Universe here)

$$
\frac{d}{d t}\left(a^{3} n\right)=a^{3} \beta_{L}^{2} \sqrt{\frac{3}{\lambda}} \frac{1}{24 \pi^{2}} X \omega^{3} .
$$

Integrating this during the matter dominated expansion (3.4) from [2] we obtain the neutrino density (saturated at late times)

$$
n=\beta_{L}^{2} \frac{\sqrt{\lambda}}{16 \sqrt{3} \pi^{2}} \xi X_{\mathrm{cr}}^{3}
$$

Note, that this does not depend on time, because rate (A.3) is proportional to $X \propto t^{-1}$. This formula should be compared to $n_{N}(X)=\frac{\beta^{2} \sqrt{\lambda}}{48 \sqrt{2} \pi \xi} X_{\mathrm{cr}}^{2} X$ from Section 5.2.2 of [2].

Thus, the total active neutrino density is trice the amount of (A.7). To check, whether this leads to preheating, we should compare the energy density in the neutrinos to those in the inflaton. To do this, we need energy density instead of the number density. Just doing the computation using occupation numbers (2.3) leads to divergent result. This is caused by discontinuous approximation to mass formula (3.8). However, for the estimate purposes we can just consider, that the average energy of created neutrinos is still given by the value in appendix $\mathrm{C}$ of $[2]$, i.e. ${ }^{8}$

$$
E \sim \frac{\omega}{4 \pi \epsilon}=\frac{1}{2} \sqrt{\frac{\lambda}{3}} X
$$

Then we have for the energy density

$$
\rho \sim \frac{\lambda \beta_{L}^{2}}{96 \pi^{2}} \xi X_{\mathrm{cr}}^{3} X
$$

Equating this with the inflaton energy density (3.7) in [2] we get

$$
\frac{\lambda \beta_{L}^{2}}{96 \pi^{2}} \xi X_{\mathrm{cr}}^{3} X=\frac{\lambda}{4} X_{\mathrm{cr}}^{2} X^{2}
$$

\footnotetext{
as follows

Then, instead of (2.3) we get

$$
m_{0}-m(t)=\frac{\frac{\beta_{L}}{2 \sqrt{2}} \sqrt{\frac{3}{2 \lambda}}}{\cosh (\sqrt{2 \lambda / 3} X \cdot t)}
$$

$$
n_{k}(t)=\frac{9 \pi \beta_{L}^{2} \omega^{2}}{32 \lambda^{2} X^{2} \cosh ^{2}\left(\sqrt{\frac{3}{2 \lambda}} \frac{\pi k_{0}}{X}\right)} \frac{\sin ^{2}\left(2 l \frac{\pi k_{0}}{\omega}\right)}{\sin ^{2}\left(\frac{\pi k_{0}}{\omega}\right)} .
$$
}

${ }^{8}$ One can avoid the divergence in the calculation of energy by smoothing the effective mass (3.8), say,

Here we obviously get an exponential energy cut-off with typical scale (A.8). 
Thus, the reheating (half energy in the relativistic active neutrinos) happens at

$$
X \sim \frac{\beta_{L}^{2}}{24 \pi^{2}} \xi X_{\mathrm{cr}}
$$

This is parametrically earlier, than (3.10) in [2]. Thus, reheating temperature is

$$
T_{r} \sim\left(\frac{10 \lambda}{27 g_{*}}\right)^{1 / 4} \frac{\beta_{L}}{2 \pi \sqrt{2 \pi}} \frac{M_{P}}{\sqrt{\xi}} .
$$

This is parametrically larger than the temperature of reheating due to renormalizable operators [2],

$$
T_{r}^{\text {renorm }} \propto \frac{M_{P}}{\xi},
$$

but for $\beta_{L} \sim 1$ it gives the numerical estimate of the same order,

$$
T_{r} \simeq 1.7 \times \beta_{L} \times 10^{14} \mathrm{GeV} \sim T_{r}^{\text {renorm }} \simeq 10^{14} \mathrm{GeV} .
$$

Above we neglected the Pauli blocking effect for the fermions. Indeed, with estimates (A.7) and (A.8) we get for the occupation numbers at reheating

$$
n_{\mathbf{k}} \frac{n}{E^{3}} \simeq 10^{3} \times \beta_{L}\left(\frac{X_{\mathrm{cr}}}{X_{r}}\right)^{3} .
$$

This reaches one (breakdown of perturbative approximation because of Pauli blocking effects) for $\beta_{L} \sim 1$ at the higher reheating temperature in [2] (see formula (3.21) there). So, the Pauli blocking effects were not really relevant for this calculation.

\section{References}

[1] F. L. Bezrukov and M. Shaposhnikov, The Standard Model Higgs boson as the inflaton, Phys. Lett. B659 (2008) 703-706, [arXiv:0710.3755], [SPIRES].

[2] F. Bezrukov, D. Gorbunov, and M. Shaposhnikov, On initial conditions for the Hot Big Bang, JCAP 0906 (2009) 029, [arXiv:0812.3622], [SPIRES].

[3] J. Garcia-Bellido, D. G. Figueroa, and J. Rubio, Preheating in the Standard Model with the Higgs-Inflaton coupled to gravity, Phys. Rev. D79 (2009) 063531, [arXiv:0812.4624], [SPIRES].

[4] A. Boyarsky, O. Ruchayskiy, and M. Shaposhnikov, The role of sterile neutrinos in cosmology and astrophysics, Ann.Rev.Nucl.Part.Sci. 59 (2009) 191-214, [arXiv:0901.0011], [SPIRES].

[5] WMAP Collaboration, E. Komatsu et. al., Five-Year Wilkinson Microwave Anisotropy Probe (WMAP) Observations:Cosmological Interpretation, Astrophys. J. Suppl. 180 (2009) 330-376, [arXiv:0803.0547], [SPIRES].

[6] F. L. Bezrukov, A. Magnin, and M. Shaposhnikov, Standard Model Higgs boson mass from inflation, Phys. Lett. B675 (2009) 88-92, [arXiv: 0812.4950], [SPIRES].

[7] F. Bezrukov and M. Shaposhnikov, Standard Model Higgs boson mass from inflation: two loop analysis, JHEP 07 (2009) 089, [arXiv: 0904.1537], [SPIRES]. 
[8] A. De Simone, M. P. Hertzberg, and F. Wilczek, Running Inflation in the Standard Model, Phys. Lett. B678 (2009) 1-8, [arXiv:0812.4946], [SPIRES].

[9] A. O. Barvinsky, A. Y. Kamenshchik, C. Kiefer, A. A. Starobinsky, and C. F. Steinwachs, Higgs boson, renormalization group, and cosmology, arXiv:0910.1041, [SPIRES].

[10] F. Bezrukov, A. Magnin, M. Shaposhnikov, and S. Sibiryakov, Higgs inflation: consistency and generalisations, JHEP 1101 (2011) 016, [arXiv:1008.5157], [SPIRES].

[11] C. P. Burgess, H. M. Lee, and M. Trott, Power-counting and the Validity of the Classical Approximation During Inflation, JHEP 09 (2009) 103, [arXiv:0902.4465], [SPIRES].

[12] J. L. F. Barbon and J. R. Espinosa, On the Naturalness of Higgs Inflation, Phys. Rev. D79 (2009) 081302, [arXiv:0903.0355], [SPIRES].

[13] S. Ferrara, R. Kallosh, A. Linde, A. Marrani, and A. Van Proeyen, Jordan Frame Supergravity and Inflation in NMSSM, Phys.Rev. D82 (2010) 045003, [arXiv:1004.0712], [SPIRES].

[14] G. F. Giudice and H. M. Lee, Unitarizing Higgs Inflation, Phys.Lett. B694 (2011) 294-300, [arXiv: 1010.1417], [SPIRES].

[15] S. Weinberg, General relativity: An einstein century survey, in General Relativity: An Einstein century survey (S. W. Hawking and W. Israel, eds.), ch. 16, p. 790. Cambridge University Press, 1979.

[16] J. Hisano, Proton decay in the supersymmetric grand unified models, in Fujiyoshida 2000, Neutrino oscillations and their origin, pp. 93-102, 2000. hep-ph/0004266.

[17] Particle Data Group Collaboration, K. Nakamura et. al., Review of particle physics, J.Phys.G G37 (2010) 075021.

[18] M. E. Shaposhnikov, Baryon Asymmetry of the Universe in Standard Electroweak Theory, Nucl. Phys. B287 (1987) 757-775, [SPIRES].

[19] M. Fukugita and T. Yanagida, Sphaleron induced baryon number nonconservation and a constraint on majorana neutrino masses, Phys.Rev. D42 (1990) 1285-1286.

[20] A. E. Nelson and S. M. Barr, Upper bound on baryogenesis scale from neutrino masses, Phys.Lett. B246 (1990) 141-143.

[21] S. Khlebnikov and M. Shaposhnikov, Melting of the Higgs vacuum: Conserved numbers at high temperature, Phys.Lett. B387 (1996) 817-822, [hep-ph/9607386], [SPRIES].

[22] T. Asaka and M. Shaposhnikov, The $\nu M S M$, dark matter and baryon asymmetry of the universe, Phys. Lett. B620 (2005) 17-26, [hep-ph/0505013], [SPRIES].

[23] A. Roy and M. Shaposhnikov, Resonant production of the sterile neutrino dark matter and fine-tunings in the $\nu$ MSM, Phys.Rev. D82 (2010) 056014, [arXiv:1006.4008], [SPIRES].

[24] M. Shaposhnikov and I. Tkachev, The $\nu M S M$, inflation, and dark matter, Phys. Lett. B639 (2006) 414-417, [hep-ph/0604236], [SPRIES].

[25] F. Bezrukov and D. Gorbunov, Light inflaton Hunter's Guide, JHEP 1005 (2010) 010, [arXiv:0912.0390], [SPIRES].

[26] S. Dodelson and L. M. Widrow, Sterile-neutrinos as dark matter, Phys. Rev. Lett. 72 (1994) 17-20, [hep-ph/9303287], [SPRIES]. 
[27] X.-D. Shi and G. M. Fuller, A new dark matter candidate: Non-thermal sterile neutrinos, Phys. Rev. Lett. 82 (1999) 2832-2835, [astro-ph/9810076], [SPRIES].

[28] M. Laine and M. Shaposhnikov, Sterile neutrino dark matter as a consequence of

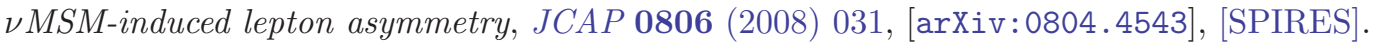

[29] A. Boyarsky, J. Lesgourgues, O. Ruchayskiy, and M. Viel, Realistic sterile neutrino dark matter with keV mass does not contradict cosmological bounds, Phys. Rev. Lett. 102 (2009) 201304, [arXiv: 0812.3256], [SPIRES].

[30] G. Bertone, W. Buchmuller, L. Covi, and A. Ibarra, Gamma-Rays from Decaying Dark Matter, JCAP 0711 (2007) 003, [arXiv:0709.2299], [SPIRES].

[31] D. Gorbunov and M. Shaposhnikov, How to find neutral leptons of the $\nu$ MSM?, JHEP 10 (2007) 015, [arXiv:0705.1729], [SPIRES]. 